\title{
Consensus control of electric spring using back-to-back converter for voltage regulation with ultra-high renewable penetration
}

\author{
Yu ZHENG ${ }^{1}$, Congchong ZHANG ${ }^{1}$, David J. HILL ${ }^{1,2}$, \\ Ke MENG'
}

\begin{abstract}
Recent advances in a power electronic device called an electric spring (ES) provide feasible solutions to meeting critical customers' requirements for voltage quality. A new version of the ES was introduced based on a back-to-back converter (ESBC) configuration which extends the operating range and improves the voltage suppression performance to facilitate ultra-high renewable penetration. This paper proposes an efficient control method to facilitate the voltage regulation function of an ESBC with non-critical loads. Particularly, the proposed method is suitable for various load characteristics. We also develop a consensus algorithm to coordinate multiple ESs for maintaining critical bus voltage in distribution systems with ultra-high renewable penetration. The proposed operation of the ESBC is verified by simulation of a modified IEEE 15-bus distribution network. The results show that the ESBC can effectively regulate system voltage and is superior to the original version of the ES.
\end{abstract}

CrossCheck date: 7 October 2017

Received: 29 April 2017/Accepted: 7 October 2017/Published online: 20 November 2017

(C) The Author(s) 2017. This article is an open access publication

$\triangle$ Yu ZHENG

zhy9639@hotmail.com

Congchong ZHANG

cczhang@eee.hku.hk

David J. HILL

dhill@eee.hku.hk

Ke MENG

ke.meng@sydney.edu.au

1 Department of Electrical and Electronic Engineering, The University of Hong Kong, Hong Kong, China

2 School of Electrical and Information Engineering, The University of Sydney, Sydney, NSW 2006, Australia
Keywords Electric spring, Back-to-back converter, Consensus control, Voltage regulation

\section{Introduction}

The increasing penetration of distributed intermittent renewable generation, such as wind and solar, has significantly challenged the operation of power grid, especially at the distribution level. One key challenge is voltage control [1-3]. Ultra high renewable penetration brings significant uncertainties in system net demand leading to sharp power fluctuations and voltage violations. The voltage regulation of current distribution systems relies mainly on conventional devices, such as on-load tap changers (OLTCs) and capacitor banks (CBs) [4]. The coordination of such conventional instruments has been widely studied to maintain the system voltage within an allowable range [5-7]. Due to the wear and tear on OLTCs if used too frequently, and to other constraints, it is difficult for these devices to meet the increased voltage regulation requirements caused by frequent and significant power fluctuation due to high renewable penetration.

Voltages in the distribution system depend on both active and reactive power due to the relatively high feeder ratio of resistance and inductance. Much existing research has addressed the voltage control problem by using distributed resources. Ye et al. apply energy storage systems for voltage regulation and peak load shaving [8]. Zeraati solves the voltage rise during the peak PV generation as well as the voltage drop while meeting the peak load with battery energy storage systems [9]. Due to the high investment cost, batteries should be designed for multiple objectives to increase their economic feasibility. Distributed reactive power control methods for distributed 
generation (DG) have been proposed [10-12]. Reference [13] investigates the effectiveness and general performance of different reactive and active power control concepts. Coordinating DG with various voltage regulators is more effective than controlling DG alone; the interactions among DG units and voltage regulating devices are identified in [14]. Ranamuka proposed a control method to coordinate voltage regulators and DG for voltage support and demonstrated the performance [15]. However, limited by their operating constraints and ownership, most DGs are still uncontrollable for the system operator (DSO).

As the renewable penetration keeps increasing, the voltage rise problem becomes greater and causes voltage regulation problems. New voltage regulation devices are essential to improve the feeder voltage profile and meet the power quality requirements of critical customers. The electric spring (ES) was introduced in [16] to implement Hooke's law for electrical systems. The device can facilitate non-critical loads to provide grid services in respect of voltage and frequency. The general performance of the ES has been demonstrated in $[17,18]$. The results verified the capability and flexibility of the ES in supporting the voltage of a bus where critical "load" is allocated. According to $[7,16]$, the term "critical" load refers to an electric load that requires a well-regulated mains voltage. However, the original version of the ES has two limitations: (1) it is better at a supporting function to prevent under-voltage than a suppressing function to prevent over-voltage; (2) its voltage regulation performance depends strongly on load characteristics. A new version of the ES with a back-to-back converter (ESBC) configuration has been designed to overcome these limitations and extend the operational flexibility [19]. The operating principle and extended operation range of this back-to-back electric spring has been explained in [20]. The key issue to facilitate it to provide an effective voltage regulation service is to decide the best operating points within the full range of its operating region.

In this paper, an efficient control method is proposed to enhance the voltage regulation capability of the ES in power distribution systems with ultra high renewable penetration. Load models and some assumptions applied in this paper have been explained in detail in [16], and this work focuses on the following aspects. First, an efficient control algorithm is proposed for the ESBC to track the load power factor. Second, a consensus algorithm is developed to coordinate multiple ESBCs to support the voltage. By using the proposed control method, the system operator can decide the operating points of the ESBC taking advantage of its flexible operating range.

This paper is organized as follows. After the introduction, the limitations of the original ES (ES-1) are illustrated and new ES model is briefly presented with load modelling.
After that, the control method for a single ESBC and the voltage regulation scheme with multiple ESBCs are presented and discussed. Then, the proposed control strategy is verified on a modified IEEE 15-bus distribution network. Conclusions and further developments are discussed in the last section.

\subsection{ESBC and smart load modelling}

In this section, a smart load model with an ESBC is studied and an efficient control method is proposed. Compared with previous research on consensus control of ES [16] and ESBC control methods [20], this work discuss the control limitations of the ES-1 and studies a new design of ES. A novel control method is proposed for an ESBC to improve the performance of smart loads and facilitate consensus control of multiple ESBCs.

\subsection{Limitations of ES-1}

The original version of an ES is considered as a purely resistive or inductive voltage source and connected with a non-critical load in series to work as a smart load. The main power circuit is composed of a full-bridge or half bridge power inverter. The AC output of the inverter is controlled by pulse-width-modulation (PWM). Through an LC filter, a controllable sinusoidal voltage source with the same frequency to the grid is generated. The phasor of output voltage is controlled to be in quadrature to the phasor of current to avoid energy consumption or generation. Therefore, the load characteristic has significant impact on the smart load's performance. In previous research, a purely resistive load has been widely used to verify the voltage regulation effect of the ES. In this experimental setting, the ES output voltage decreases the resistive load voltage in both capacitive and inductive modes. Hence, the ES-1 has better voltage-support function than voltage suppression function with a purely resistive load. This effect is not efficient in dealing with voltage problems caused by high renewable penetration where over-voltage is the major issue. The voltage vectors of the smart load are shown in Fig. 1. The operation of ES in both capacitive and inductive modes decreases the non-critical load voltage and hence reduces the load power consumption. This effect enhances the voltage support function while reducing the voltage suppression function of the ES.

Moreover, load characteristics can significantly affect the control effect of a smart load with an ES-1. We simulated the voltage regulation effects using continuous ES output with different load characteristics to illustrate the limitations of the ES-1. To show the control effects on line 


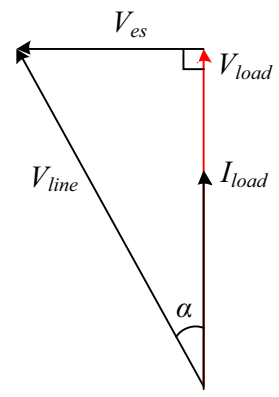

(a) Inductive mode

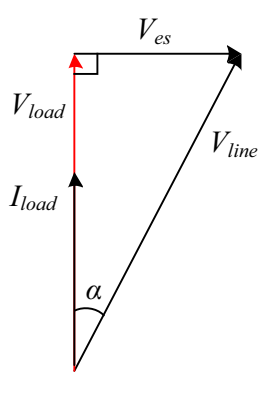

(b) Capacitive mode
Fig. 1 Voltage vectors of smart load with purely resistive load

voltage more clearly, we used a constant load with different power factors. The results are shown in Fig. 2.

As shown in Fig. 2, the control effects of the inductive and capacitive load are obviously quite different. Usually, line voltage increases as capacitive ES output increases. However, the ES control reduced the line voltage for a capacitive load in this test. We define this as a negative control effect and marked it as such in Fig. 2. In previous ES studies, researchers increase the capacitive ES output to increase the line voltage, and conversely increase the inductive ES output to reduce the line voltage. Such a control method causes a control error in this certain load scenario. An innovation with a new configuration of ES provides a solution to overcome these limitations.

\subsection{Basic concept of ESBC}

This section explains the topology of the ESBC and its operating range. The new ESBC consists of two converters in a back-to-back configuration and the basic schematic is shown in Fig. 3. One of the converters (converter 1) is used to generate series voltage sources, and the other (converter 2 ) is connected between main grid and ground to compensate the real power consumption of converter 1 . A common DC link provides a power flow channel between the two converters. Detailed hardware implementation of

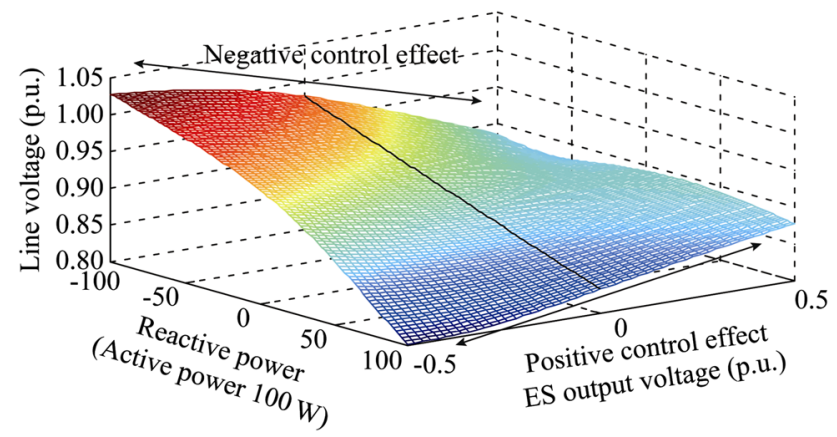

Fig. 2 Different voltage regulation effects of an ES-1 with different load characteristics

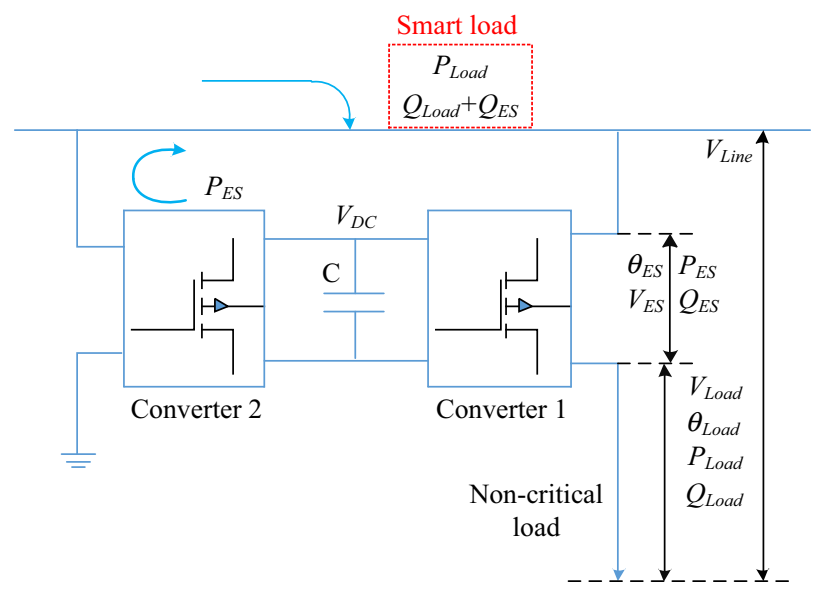

Fig. 3 Circuit diagram of ESBC

the ESBC has been explained in [20]. Converter 1 plays the same role as the ES-1 providing an adaptive voltage $\left(V_{e s}\right)$ for the non-critical load, represented by (1) and (2). Converter 2 compensates the real power consumption to maintain the DC link voltage. By adjusting the non-critical load voltage adaptively, the load can be regarded as a fast demand-response resource providing grid support. Together with the ES, the non-critical load becomes a smart load to balance fluctuations of supply and demand. Compared with the ES-1, the new version has one more control parameter, the voltage angle (see Fig. 4). ESBC operation has more flexibility and a wide range of operation, while a novel control algorithm is required to determine the new control parameter.

$V_{E S}=m \% \cdot V_{E S}^{\max }$

$\vec{V}_{E S}+\vec{V}_{\text {Load }}=\vec{V}_{\text {Line }}$

where $\vec{V}_{E S}, \vec{V}_{\text {Load }}$, and $\vec{V}_{\text {Line }}$ are the voltage vectors of the $\mathrm{ES}$, the load and the bus, respectively; $m$ is modulation index; and $V_{E S}$ and $V_{E S}^{\max }$ are the magnitude of ES output voltage and output limit.

In Fig. 3, the active and reactive power consumption of the non-critical load are denoted by $P_{\text {Load }}$ and $Q_{\text {Load }}$. The active power consumption and feed back to the grid by converter 1 and converter 2 are the same, denoted by $P_{E S}$. The reactive power consumption by the ES is represented by $Q_{E S} . \theta_{E S}$ and $\theta_{\text {Load }}$ are the power factor of the load and the ES. The total active and reactive power consumption

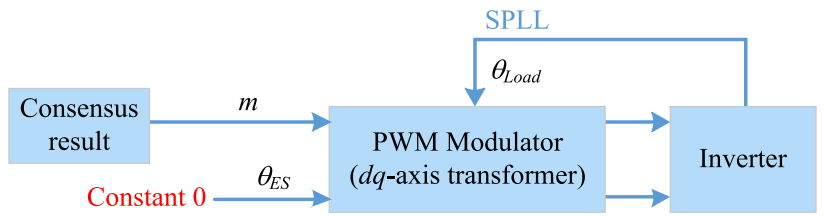

Fig. 4 Overall control scheme for single ES 
that the smart load injects to the grid are $P_{\text {Load }}$ and $Q_{\text {Load }}+Q_{E S}$.

The cost of the ES is not cheap and as such voltage regulation in this paper is implemented for the critical bus. Investment in ESs could be by the utility where there is a particular voltage regulation problem, or by customers who require high power quality. If the utility pays for the ESs, a power quality surcharge should be charged to the customers who benefit from it. Detailed incentive mechanism could be developed in future work.

\subsection{Control scheme for single ESBC}

Unlike the ES-1 which injects reactive power between a non-critical load and the power grid regardless of the voltage and frequency situation, the ESBC is designed to provide active and reactive power for a non-critical load while the active power is compensated by the grid through converter 2. Due to the two degrees of freedom now available, $V_{e s}$ and $\theta_{e s}$, it is necessary to design an efficient control strategy for system voltage support.

Therefore, a control scheme for the ESBC is proposed in this work to resolve this issue. The schematic of this control scheme is shown in Fig. 4.

For clarity, we only explain the control scheme for converter 1 is this paper. The control loop for converter 2 follows the technique in [20] to maintain stable DC voltage and balance power flow between the two converters. In the proposed control scheme, a sampled phase-locked loop (SPLL) is introduced to track the power factor of the non-critical load. The phase angle of the ES output voltage $\theta_{e s}$ is locked to the non-critical load's phase angle. In realtime operation, voltage support can be implemented by setting a positive modulation index while voltage suppression is achieved with a negative value. In this way only one control parameter, the modulation index $m$, is required to implement voltage regulation. In Figs. 5 and 6 we compare the operating region and control effect of the ES-1
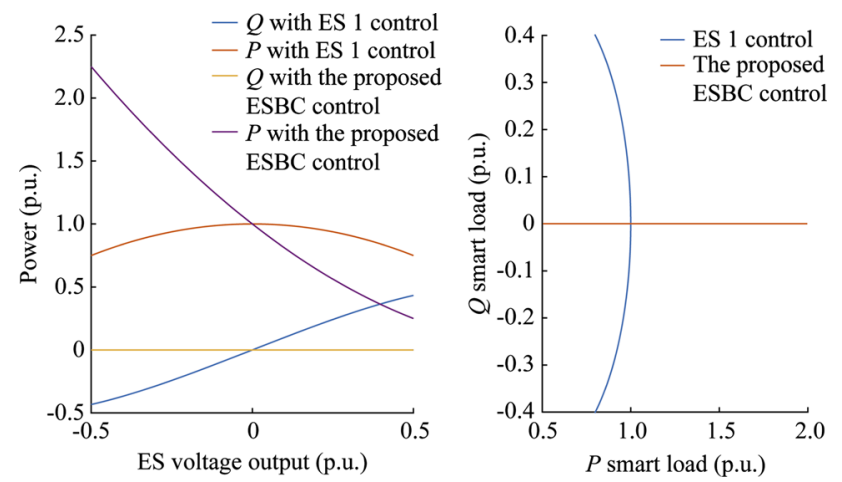

Fig. 5 Comparison of the proposed ESBC control strategy and the ES1 control method with a purely resistive load
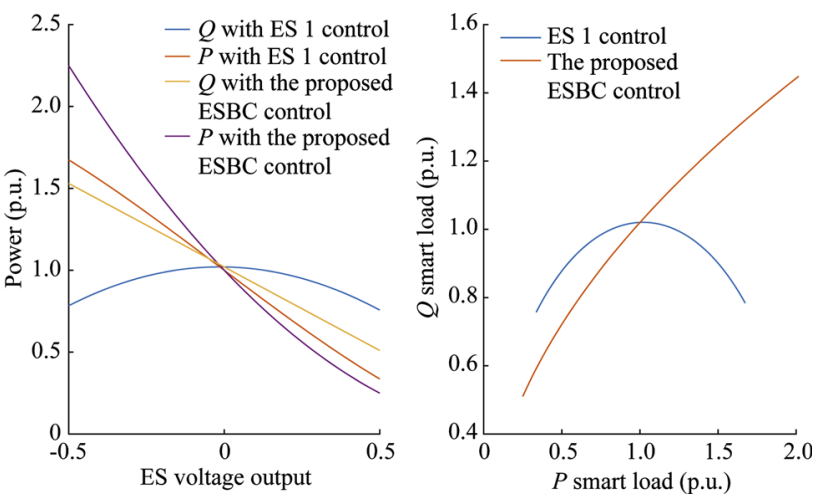

Fig. 6 Comparison of the proposed ESBC control strategy and the ES1 control method with an inductive load $(P F=0.7)$

and the ESBC with a purely resistive load and inductive load, respectively.

Compared with the ES-1, the ESBC with the proposed control scheme provides more flexible active power compensation capability within the same range of ES voltage magnitude. Reactive power compensation depends on the load characteristics and power factor. The advantages of the proposed control scheme are:

1) It is simple and convenient to implement the control scheme for voltage regulation;

2) Negative control effects shown in Fig. 2 can be avoided by using the proposed scheme;

3) The scheme provides a higher voltage regulation capability within the same operating range.

\subsection{Load model}

In this paper, we find that the operation of the ES affects the voltage of the load significantly and consequently changes the demand. In previous ES studies, only resistive and inductive demand is considered for voltage support and the control performs well. In order to extend the method more generally, load models should be considered to verify the proposed strategy. Many load models have been proposed and applied for system analysis. This paper adopts a common approach which is to consider the load in three categories: constant impedance, constant current, and constant power, referred to as a ZIP model. The active and reactive power consumption of a load are expressed by the quadratic functions given in (3) and (4). The parameters of the load can be obtained by using statistical methods or load monitoring techniques which are not discussed in detail in this paper. 
$P_{i}^{L}=P_{i}^{L, N}\left[a_{i}^{p}+b_{i}^{p}\left(\frac{V_{o, i}}{V_{N}}\right)+c_{i}^{p}\left(\frac{V_{o, i}}{V_{N}}\right)^{2}\right]$

$Q_{i}^{L}=Q_{i}^{L, N}\left[a_{i}^{q}+b_{i}^{q}\left(\frac{V_{\mathrm{o}, i}}{V_{N}}\right)+c_{i}^{q}\left(\frac{V_{\mathrm{o}, i}}{V_{N}}\right)^{2}\right]$

where $a_{i}^{p}, b_{i}^{p}, c_{i}^{p}, a_{i}^{q}, b_{i}^{q}$ and $c_{i}^{q}$ are the parameters of load at bus $i$; and $V_{\mathrm{o}, i}$ is the load voltage of bus $i$.

\section{Consensus control for multiple ESBCs}

\subsection{Coordination strategy}

A consensus algorithm is proposed for ESBCs to maintain the critical bus voltage, in which the leader initiates the voltage error as the control reference and distributes it to the follower. This is described below for a single critical bus; further work will consider multiple critical buses. For system with multiple critical buses, we can use the average voltage error of multiple critical buses as the control reference and distribute it to the ESBCs either by the proposed consensus algorithm or by using a centralized optimization method. A communication network is required to link all critical buses together.

Based on the control algorithm for a single ESBC, the procedures to coordinate multiple ESBSs for voltage support in distribution systems are presented in section, including the following steps:

Step 1: Initialization. The smart load information including voltage and current can be obtained from a local monitoring unit in real time

Step 2: Objective. In order to accurately maintain the critical bus voltage, a PI controller is used to represent the magnitude of the voltage deviation and provide a reference for the ESBCs

Step 3: Communication. This reference signal will then be sent to interconnected ESBCs through communication links, and updated in an iterative manner following the consensus control algorithm

Step 4: Operation. Once a reference signal received, each ESBC can adjust its modulation index and associated power factor in a local control loop

The proposed consensus-based structure for coordinating multiple ESBCs is shown in Fig. 7.

\subsection{Consensus algorithm}

The consensus algorithm is described in detail as follows. It is an effective way to share the voltage support contribution among the participating ESBCs. Since smart loads share the capacity payments from utilities, participating via an automated management program provides a benefit to all participants. The consensus algorithm is explained as follows. The objective of consensus control is to coordinate ESBCs to support the critical bus voltage. We set the critical bus as leader and assume that each ESBC only receives information from its neighboring ESBCs or the leader. The consensus control algorithm is realized in a continuous time frame. At time $t$, the modulation index of each ESBC is determined to generate the voltage with the same phase angle as the connected non-critical load. The modulation index vector is denoted by $\boldsymbol{M}^{t}=\left[m_{1}^{t}, m_{2}^{t}, \ldots, m_{n}^{t}\right]$, where $m_{i}^{t}$ represents the modulation index of the smart load $i$ at time $t$. These are the controlled parameters of the ESBCs.

When the magnitude of the critical bus voltage deviates from the reference value, the leader initiates the consensus algorithm using a proportional integral (PI) controller. Neglecting the communication time delay, the control reference of the voltage error of the critical bus is expressed in a discrete way as:

$\Delta u_{0}^{t}=k_{i} \cdot \int_{s=0}^{t} \Delta u_{0}^{s}+k_{p}\left(V_{C}^{t}-V_{r e f}\right)$

where $\Delta u_{0}^{t}$ is the control reference of the leader at time $t$. The communication network of the ESBCs is described by a graph $\boldsymbol{G}=(\boldsymbol{E}, \boldsymbol{A})$, where $\boldsymbol{E}$ is the set of smart loads and $\boldsymbol{A} \subseteq E \times E$ is the set of communication edges in the graph. Edge $(i, j) \in A$ if smart load $j$ can send information to smart load $i$. In this study, the communications are assumed to be bidirectional, so that if $(i, j) \in A$ then $(j, i) \in A$. We use $N_{i}$ as the set of all smart loads that can communicate with smart load $i$ and $\left|\boldsymbol{N}_{i}\right|$ is its cardinality. The adjacency matrix is defined as a $n \times n$ matrix $\boldsymbol{L}$.

The communication network's performance is strongly connected with the eigenvalues of the Laplacian matrix, especially the second smallest eigenvalue $\lambda_{2}$, which is called the algebraic connectivity of a graph [21]. Based on [22], the consensus algorithm's speed is measured by the algebraic connectivity of the network topology. $\lambda_{2}$ is relatively large for dense graphs and relatively small for sparse graphs, as noted regarding the Fiedler eigenvalue of an undirected graph [23]. Dense interactions means an agreement problem in a network is solved faster compared with a connected but sparse network, thus a large $\lambda_{2}$ provides a faster convergence speed than a small $\lambda_{2}$. We tested several typical communication networks in the case study below. In the consensus algorithm, the information state of each $\mathrm{ES}, \varepsilon_{i}^{t}$, is updated by the following equation, 
Hierarchical dispatch structure for multiple ESBCs
Inputs:

- Load characteristic

- ES constraints

- Load operation state

- Voltage signal

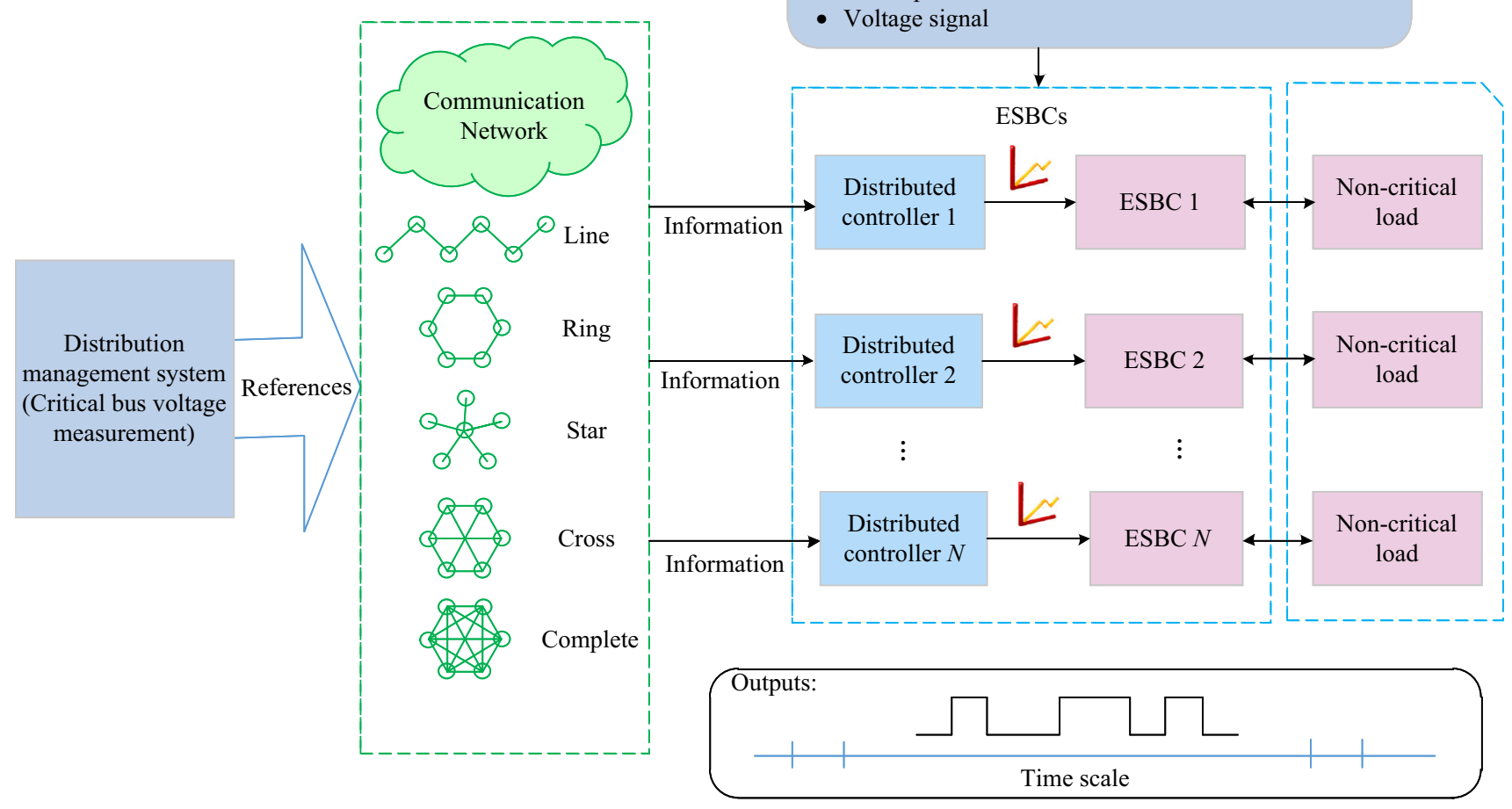

Fig. 7 Proposed hierarchical structure for dispatching multiple ESBCs

$\dot{\varepsilon}_{i}^{t}=\sum_{j \in N_{i}} d_{i j}^{t} \cdot\left(\varepsilon_{j}^{t}-\varepsilon_{i}^{t}\right) \quad i=1,2, \ldots, n$

where $d_{i j}^{t}$ is the element of a row-stochastic matrix, defined as:

$d_{i j}^{t}=\frac{\left|l_{i j}^{t}\right|}{\sum_{j \in N_{i}}\left|l_{i j}^{t}\right|}$

where $l_{i j}$ is the information flow between buses $i$ and $j$ :

$l_{i j}= \begin{cases}\sum_{i \neq j} a_{i j} & i=j \\ -a_{i j} & \text { otherwise }\end{cases}$

where $a_{i j}=1$ if $(i, j) \in A$ and 0 otherwise. This consensus algorithm is guaranteed to converge. We can also optimize the factor $l_{i j}$ to realize a fast converge speed for a large system, and this will be considered in future work. The algorithm can be written in a discrete form as:

$\varepsilon_{i}^{k+1}=\varepsilon_{i}^{k}+\alpha \sum_{j \in N_{i}} d_{i j}^{k}\left(\varepsilon_{j}^{k}-\varepsilon_{i}^{k}\right) \quad i=1,2, \ldots, n$ where $k$ is the discrete time index and $\alpha$ is the step size. The two forms of consensus algorithm above can be applied in different simulation tools used to implement the case studies below.

During the updating process, the leader ESBC acquires reference information from the critical bus and the control signals are spread out through the communication network according to the consensus algorithm. In this paper, to encourage the fair participation of all smart loads in voltage regulation, we assign the same weight to all the ESBCs. The sharing of responsibility is according to the ESBCs' regulation capability. In other words, the the ratio of voltage generation to voltage generation capabilities is kept equal among all ESBCs. We can simply set $m_{i}^{t}=$ $\varepsilon_{i}^{t} V_{E S, i}^{\max }$ for all the ESBCs to equally realize this equal sharing in the consensus state, as expressed by (10).

$\frac{m_{1}}{V_{E S, 1}^{\max }}=\frac{m_{2}}{V_{E S, 2}^{\max }}=\cdots \frac{m_{n}}{V_{E S, n}^{\max }}$

The overall process of the proposed voltage regulation method is shown in Fig. 8. 


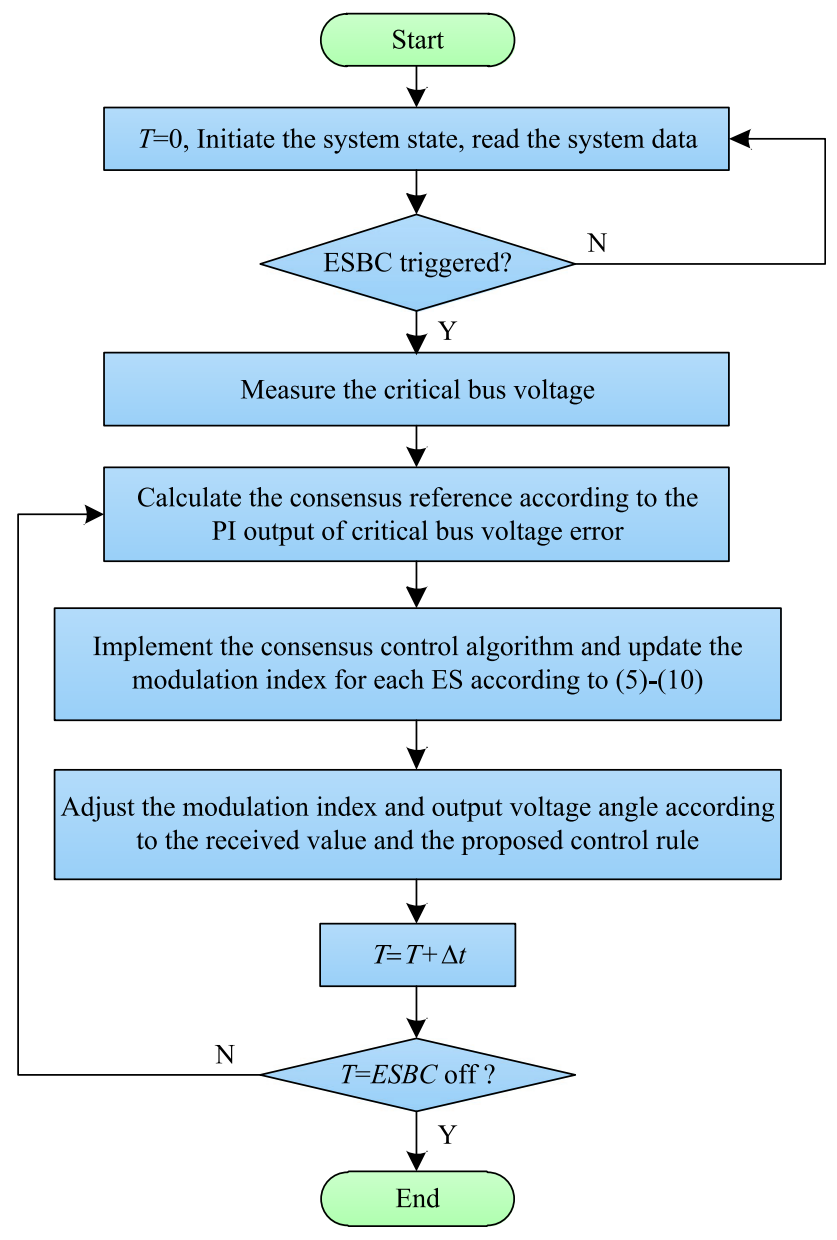

Fig. 8 Flowchart of the proposed voltage regulation method

\section{Case studies}

The proposed consensus based voltage regulation method for the ESBC has been tested on a modified IEEE15 bus distribution system and compared with the ES- 1 . The test system and the line shaped communication network are shown in Fig. 9. The rated demand is $1296 \mathrm{kWh}$ and the total capacity of distributed renewable energy is $2650 \mathrm{kWh}$. In this system, ultra-high renewable penetration causes significant voltage fluctuations where the voltage surge problem is not easily solved by original ES version and resistive loads. There are 6 smart loads, composed of ESBCs and non-critical loads, supporting a critical bus. The voltage output capacity of the ESBCs $V_{E S, i}^{\max }$ is $200 \mathrm{~V}$. Although it is shown as line shaped in Fig. 9, the communication network can be simulated with different topologies, including ring, star, cross, and complete. The ESBC located at bus 4 is designated as the virtual leader to initiate the control signal for the group of ESBCs. The system parameters are shown in Table 1.

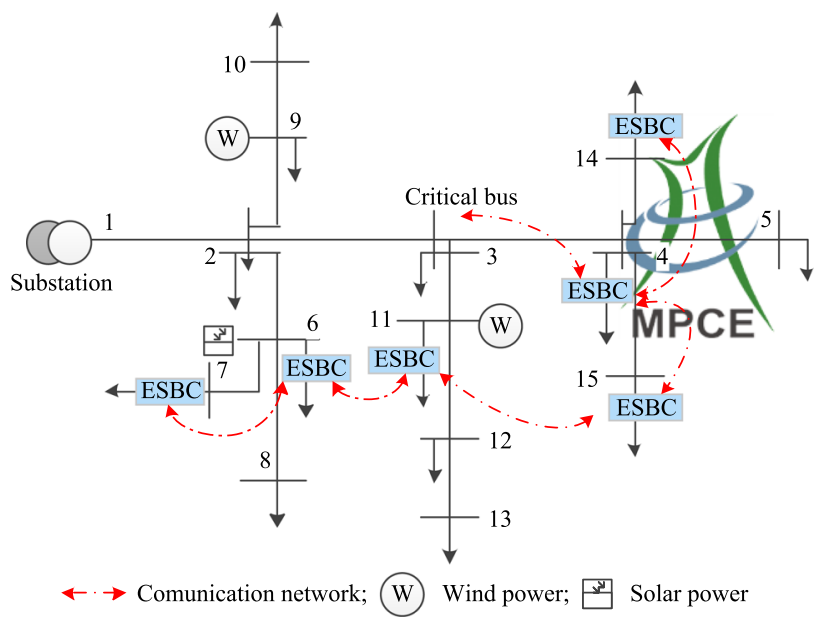

Fig. 9 Test system

Table 1 Experiment settings

\begin{tabular}{ll}
\hline Items & Value (p.u.) \\
\hline Maximum and minimum line voltage & $1.05,0.95$ \\
Maximum and minimum load voltage & $1.3,0.7$ \\
Maximum and minimum ES output & $-1,1$ \\
Preset voltage value & 0.97 \\
Proportional parameter $k_{p}$ & $5 \times 10 \mathrm{e} 6$ \\
Integral parameter $k_{i}$ & $2 \times 10 \mathrm{e} 6$ \\
$a_{i}^{p}, b_{i}^{p}, c_{i}^{p}$ & $0,0,1$ \\
$a_{i}^{q}, b_{i}^{q}, c_{i}^{q}$ & $0,0,1$ \\
\hline
\end{tabular}

\subsection{Transient response}

The first simulation is implemented in MATLAB Simulink to show the transient response of multiple ESBCs after a sudden power change event and to demonstrate the voltage regulation effect for the critical bus voltage. The simulations are performed with the ODE-45 solver and 0.001 relative tolerance. Communication time delays are not considered here. Sudden generation power changes are programmed to test the voltage support and suppression effects of the ESBCs.

The ESBCs are activated at $1 \mathrm{~s}$ to maintain the critical bus voltage at 0.97 p.u. A sudden $400 \mathrm{~kW}$ loss of generation occurs at $10 \mathrm{~s}$ and causes a voltage dip. The critical bus voltage deviation triggers the operation of ESBCs. The dynamic responses of multiple ESBCs and the critical bus voltage profile after the generation loss are shown in Fig. 10. It can be seen that the critical bus voltage can be restored to 0.97 p.u. rapidly after generation loss. The voltage support function of the ESBCs is confirmed. 

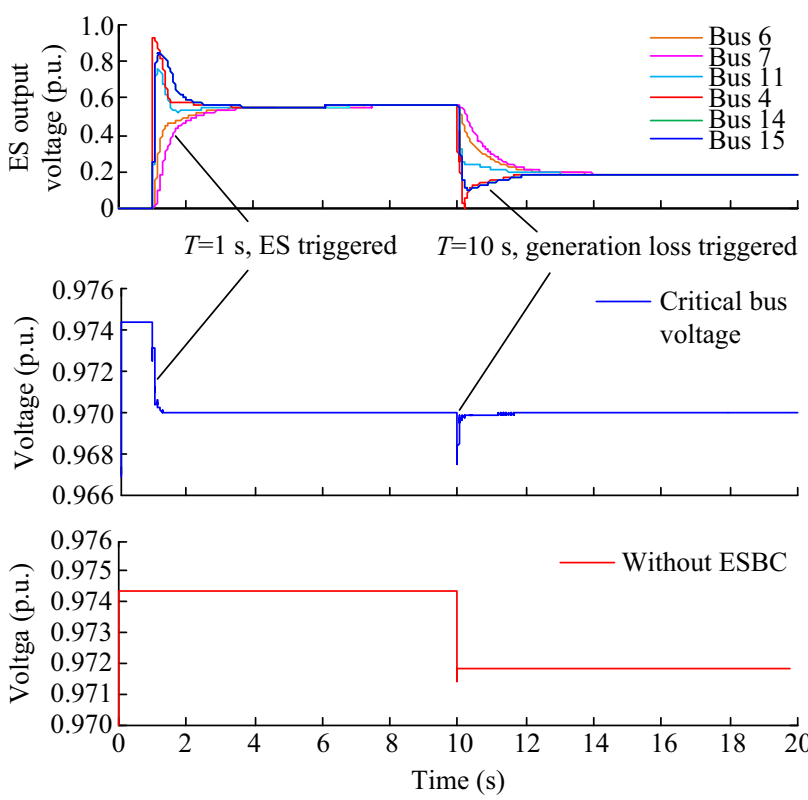

Fig. 10 Dynamic response of ESBCs and critical bus voltage during a generation loss event

Then, we test the voltage suppression effect of the proposed regulation method by programming a distributed generation increase. The ESBCs are activated at $1 \mathrm{~s}$ as in the first simulation to maintain the critical bus voltage at 0.97 p.u. and the distributed generation rise occurs at $10 \mathrm{~s}$. The power generation output is increased from 400 to $800 \mathrm{~kW}$ and causes a voltage rise. After the disturbance, the ESBCs are coordinated to regulate the critical bus voltage back to the preset value of 0.97 p.u. The dynamic responses of multiple ESBCs for voltage suppression are
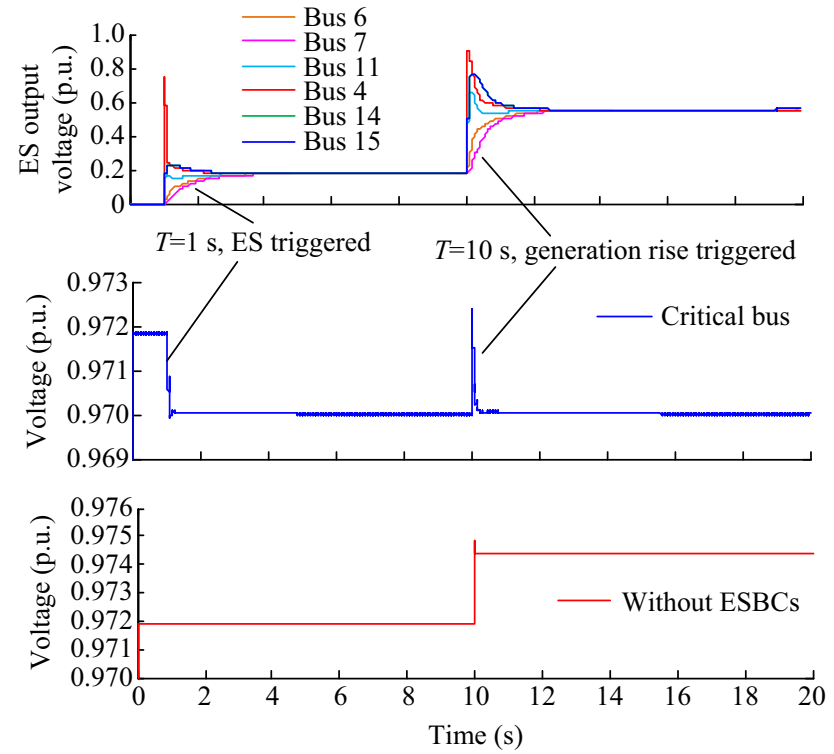

Fig. 11 Dynamic response of ESBCs and critical bus voltage during a generation increase shown in Fig. 11. It can be observed that the capability of the ESBCs in suppressing the critical bus voltage is demonstrated.

\subsection{Communication network sensitivity analysis}

Since the communication topology has impacts on the algorithm's convergence and the system dynamics, sensitivity studies are conducted in this section to show the robustness of the proposed dispatch scheme with different topologies of communication network. As shown in Fig. 12a-d, four different topologies are studied, including ring, star, cross, and complete. The results indicate that the topology has little influence on the system dynamics. However, as the system size grows, the convergence speed of the distributed algorithm becomes highly reliant on the topology of the communication network.

From the sensitivity analysis above, we can draw a conclusion that the proposed regulation method can share the contribution fairly among the ESBCs and maintain the critical bus voltage, and the system response and consensus state are largely dependent of the topology of communication network.

\subsection{Continuous voltage regulation}

In this case study, the ESBCs are simulated using MATLAB to provide continuous voltage regulation for a critical bus with variations of demand and generation. Firstly, the bus voltage profiles through the system are calculated under the base scenario without voltage regulation. In real application, conventional voltage regulation is applied to avoid the violation of feeder voltage constraints. In this work, the conventional regulation devices are not included to show the capability of the ESBCs more clearly. For clarity, we only show the critical bus voltage profile and net demand in Fig. 13.

Due to the high penetration of variable renewable energy, we find that the critical bus voltage deviates from the desired value significantly along with the fluctuation of distributed generation output. In order to maintain the critical bus voltage, we implement consensus control for the ESBCs using the proposed voltage management scheme. We select the simulation time period from 8:00 to $12: 00$, during which the critical bus voltage problem is most significant with voltage rise caused by increasing output from distributed solar generation. The voltage regulation results are shown in Fig. 14.

The critical bus voltage is regulated closely to the desired value by using the proposed voltage regulation method. Each non-critical load voltage is controlled adaptively by the output of ESBC converter 1 to make a contribution to voltage support and suppression. The bus 

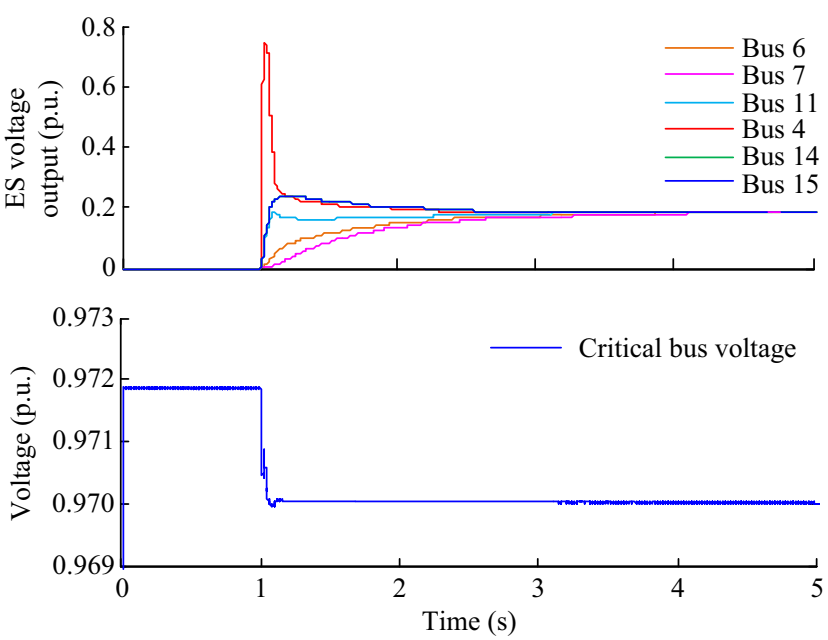

(a) Line shaped network
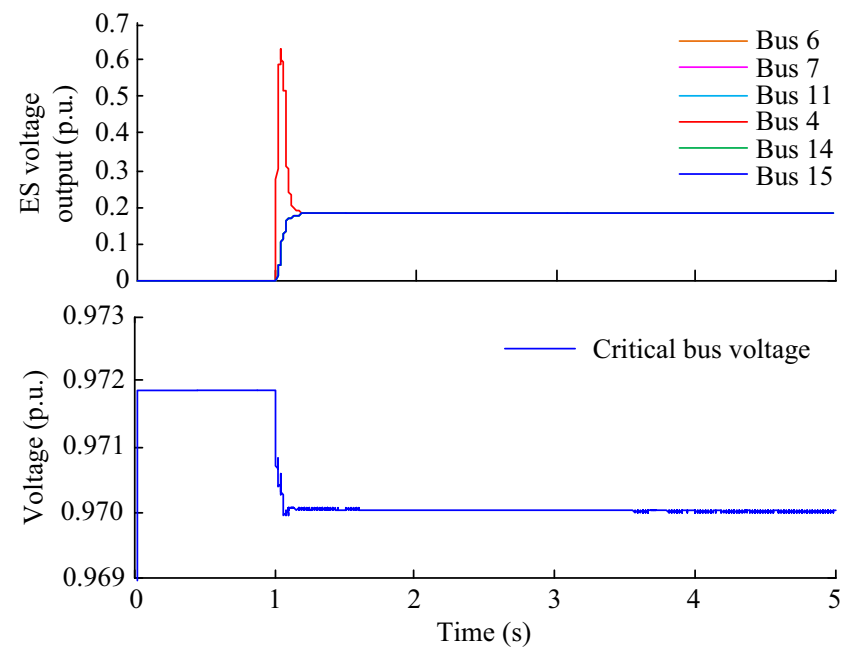

(c) Star shaped network

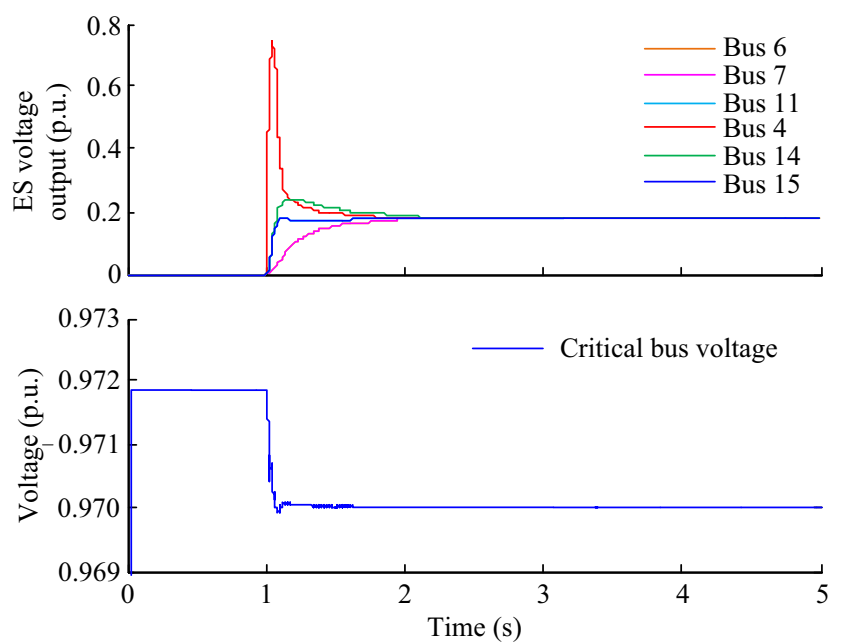

(b) Ring shaped network

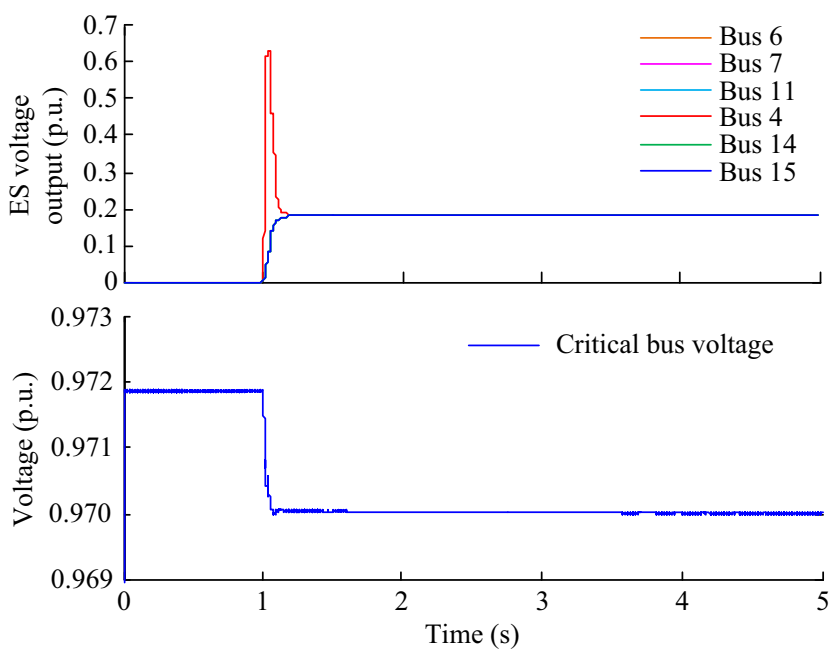

(d) Complete shaped network

Fig. 12 Results of sensitivity analysis with system dynamics under line shaped network, ring shaped network, star shaped network, and complete shaped network

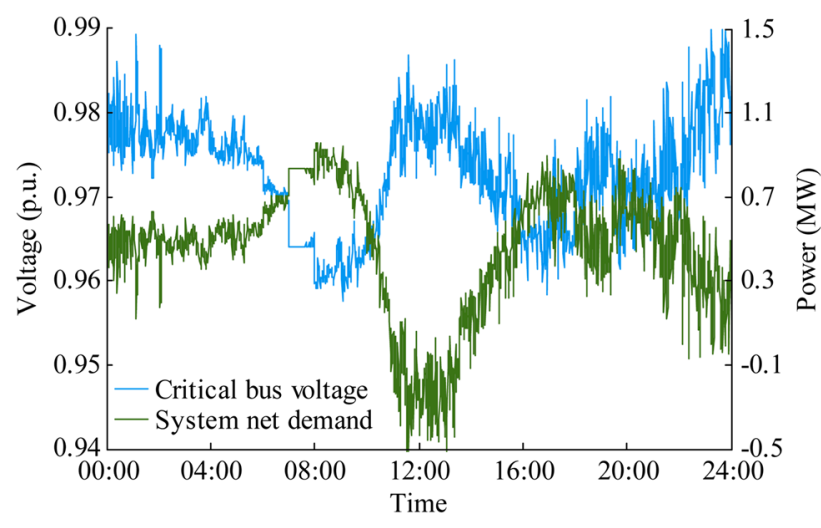

Fig. 13 Critical bus voltage profile and net demand of a typical day

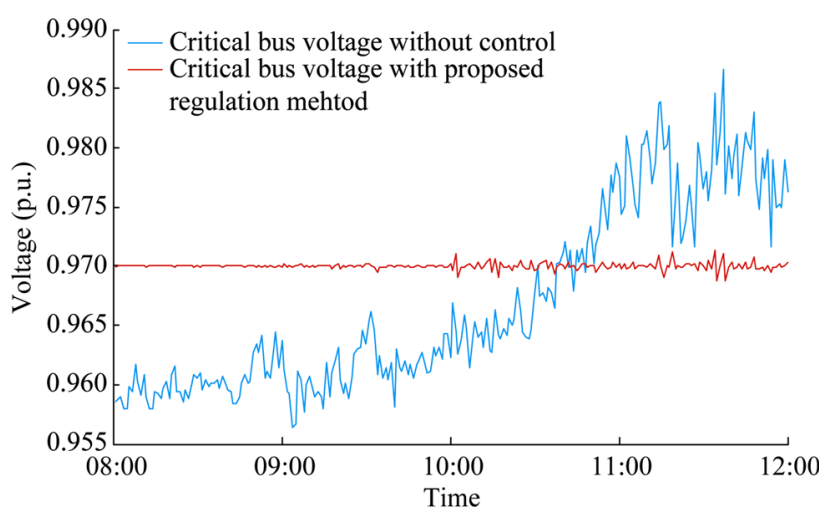

Fig. 14 Comparison of critical bus voltage with and without ESBC 


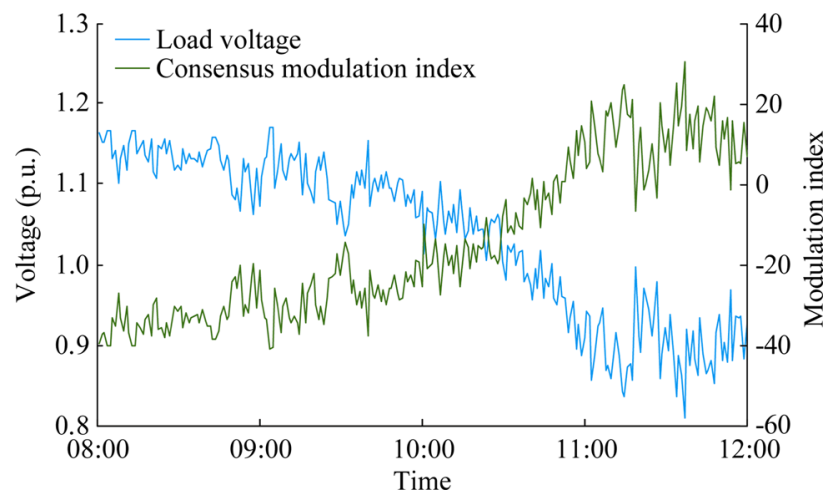

Fig. 15 Load voltage and modulation index of ESBC based smart load

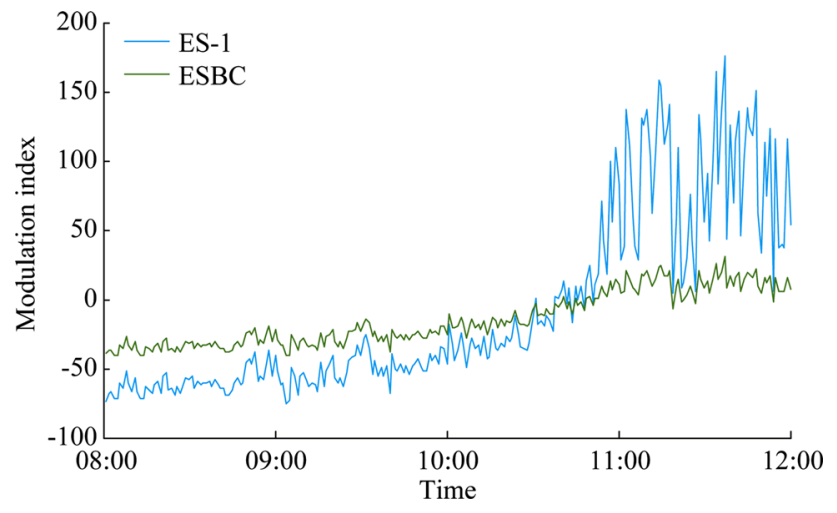

Fig. 16 Modulation index comparison of ES-1 and ESBC

voltage and the load voltage of bus 4 where the leader ESBC is located are shown in Fig. 15.

We also compare the performance of the ES- 1 and the ESBC under the same experiment settings. Both devices can achieve accurate voltage regulation. However, the operating costs are different. The comparison results are shown in Fig. 16. The voltage output of the ES-1 is much higher than that of the ESBC, especially in voltage suppression mode. In other words, the ESBC has better voltage regulation capability than the ES-1 when using the consensus control algorithm. In a system with ultra-high renewable energy penetration, the ESBC shows high performance in voltage suppression.

\section{Conclusion}

This paper studies a new version of the ES and analyzes the voltage control limitations of the original ES. The voltage regulation effect of a new ES based on an ESBC is investigated. Users with a non-critical load can place an ESBC in series with it to work as a smart load, so there is high potential for practical applications.
This paper designs a simple control rule for single ESBC and coordinates multiple ESBCs in the distribution system for critical voltage regulation by using a consensus algorithm. In the proposed control method, ESBCs are controlled to operate at the same power factor as the noncritical loads they connect. This method provides similar voltage suppression capability to the original ES, and better voltage support.

The ESBCs share the responsibility for voltage regulation, and by taking advantage of limited communication links to exchange information, they arrive at an equilibrium point for sharing the regulation contribution fairly among ESBCs.

The simulation results have verified the effectiveness of the proposed voltage regulation method and compared the regulation performance of the ESBC with the original ES. Future work will focus on decoupling analysis of ESBC for both frequency control and voltage control.

Acknowledgements The work was fully supported by a grant from the Research Grants Council of the Hong Kong Special Administrative Region under Theme-based Research Scheme through Project No. T23-701/14-N.

Open Access This article is distributed under the terms of the Creative Commons Attribution 4.0 International License (http:// creativecommons.org/licenses/by/4.0/), which permits unrestricted use, distribution, and reproduction in any medium, provided you give appropriate credit to the original author(s) and the source, provide a link to the Creative Commons license, and indicate if changes were made.

\section{References}

[1] Flocha CL, Bansalb S, Tomlinb CJ et al (2017) Plug-and-play model predictive control for load shaping and voltage control in smart grids. IEEE Trans Smart Grid. https://doi.org/10.1109/ TSG.2017.2655461

[2] Cheng D, Mather BA, Seguin R et al (2016) Photovoltaic (PV) impact assessment for very high penetration levels. IEEE J Photovolt 6(1):295-300

[3] Feng C, Li Z, Shahidehpour M et al (2017) Decentralized shortterm voltage control in active power distribution systems. IEEE Trans Smart Grid. https://doi.org/10.1109/TSG.2017.2663432

[4] Li P, Ji H, Wang C et al (2017) Coordinated control method of voltage and reactive power for active distribution networks based on soft open point. IEEE Trans Sustain Energy 8(4):1430-1442

[5] Salih SN, Chen P (2016) On coordinated control of OLTC and reactive power compensation for voltage regulation in distribution systems with wind power. IEEE Trans Power Syst 31(5):4026-4035

[6] Kulmala A, Repo S, Jarventausta P (2014) Coordinated voltage control in distribution networks including several distributed energy resources. IEEE Trans Smart Grid 5(4):2010-2020

[7] Zheng Y, Hill DJ, Meng K et al (2017) Critical bus voltage support in distribution systems with electric springs and responsibility sharing. IEEE Trans Power Syst 32(5):3584-3593 
[8] Ye Y, Hui L, Aichhorn A et al (2014) Sizing strategy of distributed battery storage system with high penetration of photovoltaic for voltage regulation and peak load shaving. IEEE Trans Smart Grid 5(1):982-991

[9] Zeraati M, Golshan MEH, Guerrero JM (2017) Distributed control of battery energy storage systems for voltage regulation in distribution networks with high PV penetration. IEEE Trans Smart Grid. https://doi.org/10.1109/TSG.2016.2636217

[10] Li H, Li F, Xu Y et al (2010) Adaptive voltage control with distributed energy resources: algorithm, theoretical analysis, simulation, and field test verification. IEEE Power Syst 25(1):1638-1647

[11] Robbins BA, Hadjicostis CN, Dominguez-Garcia AD (2013) A two-stage distributed architecture for voltage control in power distribution systems. IEEE Power Syst 28(1):1470-1482

[12] Bonfiglio A, Brignone M, Delfino F et al (2014) Optimal control and operation of grid-connected photovoltaic production units for voltage support in medium-voltage networks. IEEE Trans Sustain Energy 5(1):254-263

[13] Bletterie B, Kadam S, Bolgaryn R et al (2017) Voltage control with PV inverters in low voltage networks-in depth analysis of different concepts and parameterization criteria. IEEE Trans Power Syst 32(1):177-185

[14] Ranamuka D, Agalgaonkar AP, Muttaqi KM (2017) Examining the interactions between DG units and voltage regulating devices for effective voltage control in distribution systems. IEEE Trans Ind Appl 53(2):1485-1496

[15] Ranamuka D, Agalgaonkar AP, Muttaqi KM (2014) Online voltage control in distribution systems with multiple voltage regulating devices. IEEE Trans Sustain Energy 5(2):617-628

[16] Hui RSY, Lee CK, Wu FF (2012) Electric springs-a new smart grid technology. IEEE Trans Smart Grid 3(1):1552-1561

[17] Tan SC, Lee CK, Hui RSY (2013) General steady-state analysis and control principle of electric springs with active and reactive power compensations. IEEE Trans Power Electron 28(1):3958-3969

[18] Lee CK, Chaudhuri NR, Chaudhuri B et al (2013) Droop control of distributed electric springs for stabilizing future power grid. IEEE Trans Smart Grid 4(1):1558-1566

[19] Akhtar Z, Chaudhuri B, Hui SYR (2017) Smart loads for voltage control in distribution networks. IEEE Trans Smart Grid 8(2):937-946

[20] Yan S, Lee CK, Yang T (2017) Extending the operating range of electric spring using back-to-back converter: hardware implementation and control. IEEE Trans Power Electron 32(7):5171-5179

[21] Fiedler M (1973) Algebraic connectivity of graphs. Czechoslov Math J 23(98):298-305

[22] Olfati-Saber R, Fax J, Murray R (2004) Consensus problems in networks of agents with switching topology and time-delays. IEEE Trans Autom Control 49(9):1520-1533

[23] Godsil C, Royle G (2001) Algebraic graph theory (Graduate Texts in Mathematics), 1st edn. Springer, New York
Yu ZHENG (M'15) received his B.E and Ph.D degrees from Shanghai Jiao Tong University, China and The University of Newcastle, Australia, in 2009 and 2015, respectively. He is now a senior research assistant at the University of Hong Kong, Hong Kong. His research interests include power electronic applied in power system, power system planning, and smart grid.

Congchong ZHANG received the B.S. degree from Xi'an Jiaotong University, Xi' an, China, in 2014. He is currently pursuing the Ph.D. degree at the Department of Electrical and Electronic Engineering, The University of Hong Kong, Pokfulam, Hong Kong. His current research interests include power systems frequency control, distributed control, granular control and demand response.

David J. HILL (S'72-M'76-SM'91-F'93-LF'14) received the Ph.D. degree in electrical engineering from the University of Newcastle, Australia, in 1976. He holds the Chair of electrical engineering in the Department of Electrical and Electronic Engineering, University of Hong Kong, Hong Kong. He is also a part-time Professor of electrical engineering in the University of Sydney, Australia. During 2005-2010, he was an Australian Research Council Federation Fellow at the Australian National University. Since 1994, he has held various positions at the University of Sydney, Australia, including the Chair of Electrical Engineering until 2002 and again during 2010-2013 along with an ARC Professorial Fellowship. He has also held academic and substantial visiting positions at the universities of Melbourne, California (Berkeley), Newcastle (Australia), Lund (Sweden), Munich, and in Hong Kong (City and Polytechnic). His general research interests include control systems, complex networks, power systems, and stability analysis. His work is now mainly on control and planning of future energy networks and basic stability and control questions for dynamic networks. Prof. Hill is a Fellow of the Society for Industrial and Applied Mathematics, Philadelphia, PA, USA, the Australian Academy of Science, and the Australian Academy of Technological Sciences and Engineering. He is also a Foreign Member of the Royal Swedish Academy of Engineering Sciences.

Ke MENG (M'10) received Ph.D. from the University of Queensland, Australia in 2009. He is currently with the School of Electrical and Information Engineering, The University of Sydney, Australia. Before that, he was a research academic with the center for intelligent electricity networks, the University of Newcastle, Australia. He is also a visiting Professor of the Changsha University of Science and Technology. His research interest includes pattern recognition, power system stability analysis, wind power, and energy storage. 